\title{
Selection of Energy Conservation Measures in Building Design Phases Considering Level of Details
}

\author{
Mina Choi, Gahee Kim, and Sean Hay Kim
}

\begin{abstract}
Building energy simulation plays an important role in the design process by predicting building performance. Yet practitioner designers often feel frustrated during preparing simulations, if they are not sure of design variables of an Energy Conservation Measures (ECMs) requiring accurate information about building and systems. To help practitioners, this paper provides a guideline to select ECMs, evaluation simulation tools, and detailed inputs for modeling of ECM at each building design phase as a format of Level of Detail (LOD).
\end{abstract}

Index Terms-Energy conservation measure, level of detail, energy simulation, building modeling guideline.

\section{BACKGROUND AND OBJECTIVE}

A global warming caused by an increasing use of fossil fuels begins to cause a serious environmental problem. Buildings take up to $30 \%$ of national energy consumption as in lighting, electrical equipment, heating ventilating and air conditioning (HVAC) system, and refrigeration systems. To effectively and efficiently regulate building energy use, it is important to select appropriate Energy Conservation Measures (ECMs) in building design process, rather than to add some actions after construction. In a design stage, building energy simulation is a useful tool to analyze the energy performance of a building model containing ECMs. Most energy simulations, however, require an expert level of system knowledge as well as simulation knowledge. It is, thus, hard for practitioners to actively employ building energy simulations during design process.

\section{RESEARCH PROCESS}

The aim of this study is to suggest a guideline that improves a use of energy simulations in each design phase. This study proposes what simulations are appropriate to capture features of ECMs and when Level of Detail (LOD) of each ECM starts being discussed and confirmed in the design process. Fig. 1 briefly elaborates how this study has proceeded.

Manuscript received November 18, 2016; revised May 27, 2017. This work was supported by Basic Science Research Program through the National Research Foundation of Korea (NRF) funded by the Ministry of Science, ICT \& Future Planning (NRF-2015R1C1A2A01053559).

The authors are with School of Architecture, Seoul National University of Science and Technology, Seoul, South Korea (e-mail: chmina94@naver.com,_rkgm19408@naver.com, seanhay.kim@seoultech.ac.kr).
First, we have examined major tasks at each design phase and formulated a basic framework of the design process based on interviews with design engineers. Next, we have explored literature and selected ECMs available in the market and then classified them into passive measures applied to a building and Mechanical Electronic and Plumbing (MEP) measures. In the third step, LOD of each ECM has been factorized and then analyzed in which design phase the LOD can be decided. Lastly major simulation tools that have a sufficient capability of evaluating ECMs in each design stage in terms of algorithm and usability have been investigated. The final artifact of this study is well described in Table I. Readers can find a useful information concerning a choice of ECMs, evaluation tools, and information availability of the ECM at each design phase.

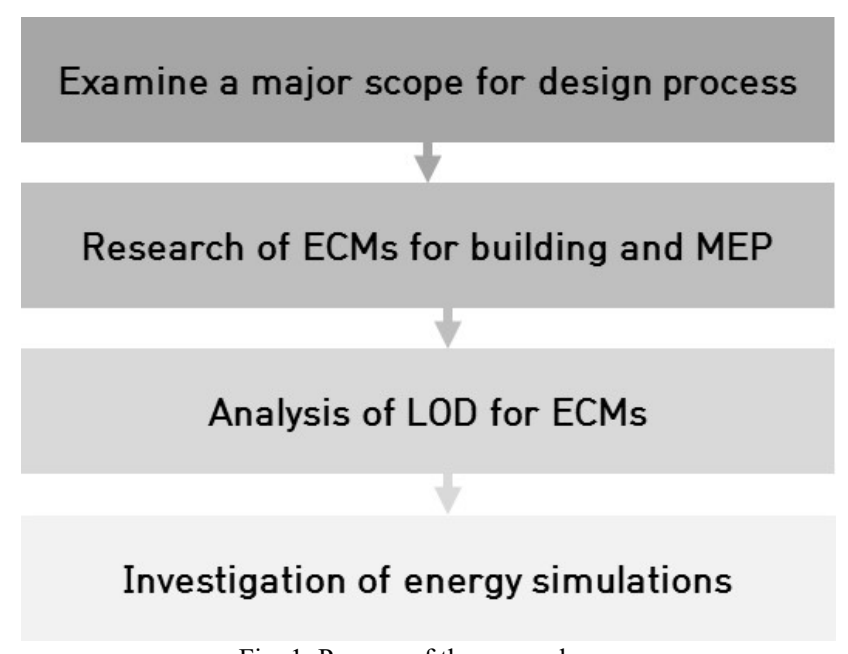

Fig. 1. Process of the research.

\section{BuILDING DESIGN PHASES}

The building design process can be divided into phases in Table II. Also, it elaborates primary tasks of each design stage in order to identify when LOD starts to be discussed and confirmed.

\section{EnERgy CONSERVATION MEASURE (ECM)}

Factors affecting energy consumption of a building can be divided into architecture, MEP, and controls as shown in Fig. 2. Architectural design can be classified into mass and layout plan, building envelope and materials. MEP design can be classified into heat source, air conditioning system, lighting system, renewable energy system, equipment. Controls mean an operation method of buildings and equipment such as scheduled ventilation and night purge that means ventilating. Most ECMs in this paper were selected based on [1]-[7]. 
TABLE I: LEVEl of DetAil (LOD) FOR PRIMARY ECMS OF BUILDINGS AND SimULATION TOOLS TO EVALUATE ECMS

\begin{tabular}{|c|c|c|c|c|c|c|c|c|c|}
\hline ECM & Simulation & Object & $\begin{array}{c}\text { Sub- } \\
\text { Object }\end{array}$ & LOD & PS & SD & $\begin{array}{l}\mathrm{D} \\
\mathrm{D}\end{array}$ & $\mathrm{CD}$ & $\begin{array}{l}\text { Cons/ } \\
\text { Occp. }\end{array}$ \\
\hline \multirow[b]{2}{*}{$\begin{array}{l}\text { Shading and daylighting } \\
\text { optimized by } \\
\text { surroundings }\end{array}$} & \multirow[b]{2}{*}{$\begin{array}{l}\text { EnergyPlus, } \\
\text { eQUEST, } \\
\text { TRNSYS }\end{array}$} & Mass & G\&T & $\begin{array}{l}\text { Placement, Area, Volume, Floor, } \\
\text { Height, Orientation }\end{array}$ & $\square$ & & & & \\
\hline & & Site & Microclimate & $\begin{array}{l}\text { Shade by surroundings, Reflection by } \\
\text { surroundings, Solar radiation } \\
\text { modulated by surroundings, Ground } \\
\text { reflectance modulated by } \\
\text { surroundings, Air temperature } \\
\text { elevation by urban heat island effect }\end{array}$ & $\square$ & & & & \\
\hline $\begin{array}{l}\text { Building mass } \\
\text { minimizing envelope area } \\
\text { and taking advantage of } \\
\text { solar gain and heat loss }\end{array}$ & $\begin{array}{l}\text { EnergyPlus, } \\
\text { eQUEST }\end{array}$ & Mass & G\&T & $\begin{array}{l}\text { Placement, Area, Volume, Floor, } \\
\text { Height, Orientation }\end{array}$ & $\square$ & & & & \\
\hline \multirow[t]{2}{*}{$\begin{array}{l}\text { Landscaping per } \\
\text { orientation considering } \\
\text { seasonal solar gain and } \\
\text { shading }\end{array}$} & \multirow[t]{2}{*}{$\begin{array}{l}\text { EnergyPlus, } \\
\text { eQUEST }\end{array}$} & Site & Microclimate & $\begin{array}{l}\text { Shade by surroundings, Reflection by } \\
\text { surroundings, Solar radiation } \\
\text { modulated by surroundings, Ground } \\
\text { reflectance modulated by } \\
\text { surroundings, Air temperature } \\
\text { elevation by urban heat island effect }\end{array}$ & $\square$ & & & & \\
\hline & & Landscape & Tree & Type, Orientation & & & $\square$ & & $\square$ \\
\hline ECM & Simulation & Object & $\begin{array}{c}\text { Sub- } \\
\text { Object }\end{array}$ & LOD & PS & SD & $\begin{array}{l}\mathrm{D} \\
\mathrm{D}\end{array}$ & $\mathrm{CD}$ & $\begin{array}{l}\text { Cons/ } \\
\text { Ocup }\end{array}$ \\
\hline \multirow{6}{*}{$\begin{array}{l}\text { Envelope finish } \\
\text { considering solar } \\
\text { absorption and } \\
\text { reflectance }\end{array}$} & \multirow{6}{*}{$\begin{array}{l}\text { EnergyPlus, } \\
\text { eQUEST, } \\
\text { TRNSYS }\end{array}$} & \multirow[b]{2}{*}{ Roof } & G\&T & Placement, Area & $\square$ & & & & $\square$ \\
\hline & & & $\begin{array}{l}\text { Exterior } \\
\text { finish }\end{array}$ & Color, Reflectance & & & $\square$ & & \\
\hline & & \multirow{4}{*}{$\begin{array}{l}\text { Window/ } \\
\text { Curtain wall/ } \\
\text { Sky light }\end{array}$} & G\&T & Placement, Area & $\square$ & & & & \\
\hline & & & Glazing & SHGC, VT & & $\square$ & & & $\square$ \\
\hline & & & $\begin{array}{l}\text { Frame/ } \\
\text { Mullion }\end{array}$ & Glass-Frame ratio & & $\square$ & & & \\
\hline & & & Shade & Exterior horizontal/vertical shade & & $\square$ & & & $\square$ \\
\hline \multirow[b]{2}{*}{ Cool roof } & \multirow{2}{*}{$\begin{array}{l}\text { EnergyPlus, } \\
\text { eQUEST, } \\
\text { TRNSYS }\end{array}$} & \multirow[b]{2}{*}{ Roof } & G\&T & Placement, Area & $\square$ & & & & $\square$ \\
\hline & & & $\begin{array}{l}\text { Exterior } \\
\text { finish }\end{array}$ & Color, Reflectance & & & $\square$ & & \\
\hline \multirow{3}{*}{$\begin{array}{l}\text { Glazing considering } \\
\text { solar gain and shading }\end{array}$} & \multirow{3}{*}{$\begin{array}{l}\text { EnergyPlus, } \\
\text { eQUEST, } \\
\text { TRNSYS }\end{array}$} & \multirow{3}{*}{$\begin{array}{l}\text { Window/ } \\
\text { Curtain wall/ } \\
\text { Sky light }\end{array}$} & Glazing & U-value, SHGC, VT & & $\square$ & & & $\square$ \\
\hline & & & $\begin{array}{l}\text { Frame/ } \\
\text { Mullion }\end{array}$ & $\begin{array}{l}\text { U-value, Glass-Frame ratio, Air } \\
\text { tightness }\end{array}$ & & $\square$ & & & \\
\hline & & & Shade & Exterior horizontal/vertical shade & & $\square$ & & & \\
\hline Electrochromic Glazing & $\begin{array}{l}\text { EnergyPlus, } \\
\text { eQUEST }\end{array}$ & $\begin{array}{c}\text { Window/ } \\
\text { Curtain wall/ } \\
\text { Sky light } \\
\end{array}$ & Glazing & SHGC, VT & & $\square$ & & & \\
\hline \multirow{3}{*}{$\begin{array}{l}\text { Air-tight envelope to } \\
\text { reduce infiltration }\end{array}$} & \multirow{3}{*}{$\begin{array}{l}\text { EnergyPlus, } \\
\text { eQUEST, } \\
\text { TRNSYS }\end{array}$} & Exterior wall & Construction & Air tightness & & $\square$ & & & \\
\hline & & Door & Vestibule & Placement, Volume & & $\square$ & & & \\
\hline & & $\begin{array}{c}\text { Window/ } \\
\text { Curtain wall/ } \\
\text { Sky light } \\
\end{array}$ & $\begin{array}{l}\text { Frame/ } \\
\text { Mullion }\end{array}$ & Air tightness & & $\square$ & & & \\
\hline \multirow{2}{*}{$\begin{array}{l}\text { Double skin facade/air } \\
\text { flow window }\end{array}$} & \multirow{2}{*}{$\begin{array}{l}\text { EnergyPlus, } \\
\text { TRNSYS }\end{array}$} & $\begin{array}{c}\text { Window/ } \\
\text { Curtain wall/ } \\
\text { Sky light }\end{array}$ & Glazing & U-value, SHGC, VT & & $\square$ & & & \\
\hline & & Exterior wall & $\begin{array}{l}\text { Frame/ } \\
\text { Mullion } \\
\end{array}$ & $\begin{array}{l}\text { U-value, Glass-Frame } \\
\text { ratio, Air tightness }\end{array}$ & & $\square$ & & & \\
\hline \multirow{3}{*}{ Green roof } & \multirow{3}{*}{$\begin{array}{l}\text { EnergyPlus, } \\
\text { TRNSYS } \\
\text { *not yet } \\
\text { commercial }\end{array}$} & \multirow{2}{*}{ Roof } & Construction & R-value, Heat capacity & & $\square$ & & & \\
\hline & & & Insulation & Insulation, R-value & & $\square$ & & & $\square$ \\
\hline & & Landscape & Tree, Grass & Type, Orientation & & $\square$ & & & $\square$ \\
\hline \multirow{3}{*}{ Green wall } & \multirow{3}{*}{ TRNSYS } & \multirow{2}{*}{ Exterior wall } & Construction & R-value, Heat capacity & & $\square$ & & & $\square$ \\
\hline & & & Insulation & R-value & & $\square$ & & & $\square$ \\
\hline & & Landscape & Grass & Type, Orientation & & $\square$ & & & $\square$ \\
\hline \multirow{2}{*}{ Light shelf } & \multirow{2}{*}{$\begin{array}{l}\text { EnergyPlus, } \\
\text { Equest }\end{array}$} & \multirow{4}{*}{$\begin{array}{l}\text { Window/ } \\
\text { Sky light }\end{array}$} & G\&T & Placement, Area & $\square$ & & & & $\square$ \\
\hline & & & Glazing & U-value, SHGC, VT & & $\square$ & & & $\square$ \\
\hline Sky light & $\begin{array}{c}\text { EnergyPlus, } \\
\text { eQUEST, } \\
\text { TRNSYS } \\
\end{array}$ & & $\begin{array}{l}\text { Frame/ } \\
\text { Mullion }\end{array}$ & $\begin{array}{l}\text { U-value, Glass-Frame } \\
\text { ratio, Air tightness }\end{array}$ & & $\square$ & & & \\
\hline Light duct & EnergyPlus & & Shade & $\begin{array}{l}\text { Exterior horizontal/ } \\
\text { vertical shade }\end{array}$ & & $\square$ & & & \\
\hline
\end{tabular}




\begin{tabular}{|c|c|c|c|c|c|c|c|c|c|}
\hline & & & Blind/Curtain & Indoor blind & & & & $\square$ & $\mathbf{\square}$ \\
\hline \multirow{3}{*}{ Trombe wall } & \multirow{3}{*}{$\begin{array}{l}\text { EnergyPlus, } \\
\text { eQUEST,T } \\
\text { RNSYS }\end{array}$} & Mass & G\&T & $\begin{array}{l}\text { Placement, Area, } \\
\text { Orientation }\end{array}$ & $\square$ & & & & \\
\hline & & Exterior wall & Construction & R-value, Heat capacity & & $\square$ & & & \\
\hline & & $\begin{array}{l}\text { Window/ } \\
\text { Curtain wall/ } \\
\text { Sky light } \\
\end{array}$ & Glazing & U-value, SHGC & & $\square$ & & & \\
\hline $\begin{array}{c}\text { EVB } \\
\text { (Exterior Venetian } \\
\text { Blinds) } \\
\end{array}$ & $\begin{array}{l}\text { EnergyPlus, } \\
\text { eQUEST,T } \\
\text { RNSYS } \\
\end{array}$ & $\begin{array}{c}\text { Window/ } \\
\text { Curtain wall/ } \\
\text { Sky light } \\
\end{array}$ & Shade & $\begin{array}{l}\text { Exterior horizontal/ } \\
\text { vertical shade }\end{array}$ & & $\square$ & & & \\
\hline \multirow{3}{*}{$\begin{array}{c}\text { PCM } \\
\text { (Phase Change Material) } \\
\text { applied in wall and } \\
\text { ceiling } \\
\end{array}$} & \multirow{3}{*}{$\begin{array}{l}\text { EnergyPlus, } \\
\text { TRNSYS }\end{array}$} & \multirow{2}{*}{ Exterior wall } & Construction & $\begin{array}{l}\text { R-value, Heat capacity, } \\
\text { Air tightness }\end{array}$ & & $\square$ & & & \\
\hline & & & Insulation & R-value & & $\square$ & & & $\square$ \\
\hline & & Ceiling & Construction & Color, Heat capacity & & $\square$ & & & $\square$ \\
\hline ECM & Simulation & Object & $\begin{array}{c}\text { Sub- } \\
\text { Object }\end{array}$ & LOD & PS & $\mathrm{SD}$ & $\begin{array}{l}\mathrm{D} \\
\mathrm{D}\end{array}$ & $\mathrm{CD}$ & $\begin{array}{l}\text { Cons/ } \\
\text { Ocup }\end{array}$ \\
\hline \multirow{4}{*}{ High efficiency plant } & \multirow{4}{*}{$\begin{array}{l}\text { EnergyPlus, } \\
\text { eQUEST, } \\
\text { TRNSYS }\end{array}$} & \multirow{2}{*}{$\begin{array}{l}\text { Heat } \\
\text { exchanger } \\
\text { for district } \\
\text { heating } \\
\end{array}$} & & Placement, Capacity & & & $\square$ & & \\
\hline & & & & Efficiency, Heat source type & $\square$ & & & & \\
\hline & & \multirow{2}{*}{$\begin{array}{l}\text { Water/Steam } \\
\text { boliler }\end{array}$} & & Placement, Heating capacity & & & $\square$ & & \\
\hline & & & & Fuel type, Tube type, Efficiency & & & & $\square$ & \\
\hline \multirow{8}{*}{$\begin{array}{l}\text { High efficiency } \\
\text { refrigerator }\end{array}$} & \multirow{8}{*}{$\begin{array}{l}\text { EnergyPlus, } \\
\text { eQUEST, } \\
\text { TRNSYS }\end{array}$} & \multirow{2}{*}{$\begin{array}{l}\text { Vapor } \\
\text { compression } \\
\text { chiller }\end{array}$} & & Placement, Capacity & & & $\square$ & & \\
\hline & & & & $\begin{array}{l}\text { COP, Compression refrigeration type, } \\
\text { Compressor type, IPLV, Performance } \\
\text { curve, Compressor control }\end{array}$ & & & $\square$ & & \\
\hline & & \multirow[b]{2}{*}{$\begin{array}{l}\text { Absorption } \\
\text { chiller }\end{array}$} & & $\begin{array}{l}\text { Placement, Cooling capacity, Hot } \\
\text { water capacity }\end{array}$ & & & $\square$ & & \\
\hline & & & & $\begin{array}{l}\text { Heat source connection } \\
\text { Fuel for direct fire } \\
\text { External heat source, } \\
\text { Cooling COP, Heating COP, IPLV, } \\
\text { Performance curve }\end{array}$ & & & & $\square$ & \\
\hline & & \multirow[b]{2}{*}{ Ice storage } & & Placement, Heat capacity & & & $\square$ & & \\
\hline & & & & $\begin{array}{l}\text { Volume, Ice making type, Insulation, } \\
\text { Refrigerant type }\end{array}$ & & & & $\square$ & \\
\hline & & \multirow{2}{*}{$\begin{array}{l}\text { CHW } \\
\text { storage }\end{array}$} & & Placement, Heat capacity & & & $\square$ & & \\
\hline & & & & Volume, Insulation & & & & $\square$ & \\
\hline \multirow{3}{*}{$\begin{array}{l}\text { Variable speed } \\
\text { compressor, condenser, } \\
\text { pump, fan }\end{array}$} & \multirow{3}{*}{$\begin{array}{l}\text { EnergyPlus, } \\
\text { eQUEST, } \\
\text { TRNSYS }\end{array}$} & \multirow{2}{*}{$\begin{array}{l}\text { Heat pump/ } \\
\text { Variable } \\
\text { Refrigerant } \\
\text { Flow (VRF) } \\
\end{array}$} & Compressor & Speed control & & & & $\square$ & \\
\hline & & & Fan & Flow control & & & & $\square$ & \\
\hline & & $\begin{array}{c}\text { Vapor } \\
\text { compression } \\
\text { chiller }\end{array}$ & & $\begin{array}{l}\text { Compression refrigerator type, } \\
\text { Compressor type, Compressor control }\end{array}$ & & & & $\square$ & \\
\hline & & & & $\begin{array}{l}\text { Placement, Cooling capacity, Hot } \\
\text { water capacity }\end{array}$ & & & $\square$ & & \\
\hline Absorption chiller-heater & $\begin{array}{l}\text { EnergyPlus, } \\
\text { eQUEST, } \\
\text { TRNSYS }\end{array}$ & $\begin{array}{l}\text { Absorption } \\
\text { chiller }\end{array}$ & & $\begin{array}{l}\text { Heat source connection } \\
\text { Fuel for direct fire } \\
\text { External heat source, Cooling COP, } \\
\text { Heating COP, IPLV, Performance } \\
\text { curve }\end{array}$ & & & & $\square$ & \\
\hline \multirow{3}{*}{$\begin{array}{l}\text { District heating and } \\
\text { cooling }\end{array}$} & \multirow{3}{*}{$\begin{array}{l}\text { EnergyPlus, } \\
\text { eQUEST, } \\
\text { TRNSYS }\end{array}$} & \multirow{3}{*}{$\begin{array}{l}\text { Heat } \\
\text { exchanger }\end{array}$} & & Placement, Capacity & & & $\square$ & & $\square$ \\
\hline & & & & Efficiency & & & & $\square$ & 口 \\
\hline & & & & Heat source type & $\square$ & & & & \\
\hline \multirow{2}{*}{$\begin{array}{l}\text { Optimal on/off for plants } \\
\begin{array}{l}\text { On-demand operation for } \\
\text { plants }\end{array}\end{array}$} & \multirow{2}{*}{$\begin{array}{l}\text { EnergyPlus, } \\
\text { eQUEST, } \\
\text { TRNSYS }\end{array}$} & \multirow{2}{*}{$\begin{array}{l}\text { Water/Steam } \\
\text { boiler }\end{array}$} & & HW reset, On-demand control & & & & $\square$ & \\
\hline & & & & On-demand control & & & & $\square$ & \\
\hline \multirow{4}{*}{$\begin{array}{l}\text { Outside air and load reset } \\
\text { for } \mathrm{CHW}, \mathrm{CW}, \mathrm{HW}\end{array}$} & \multirow{4}{*}{$\begin{array}{l}\text { EnergyPlus, } \\
\text { eQUEST, } \\
\text { TRNSYS }\end{array}$} & \multirow[b]{2}{*}{$\begin{array}{l}\text { CHW, CW, } \\
\text { HW Pump }\end{array}$} & & Placement & & & $\square$ & & $\square$ \\
\hline & & & & $\begin{array}{l}\text { Efficiency, Performance curve (Flow } \\
\text { rate, Head) } \\
\text { Flow control, Power }\end{array}$ & & & & $\square$ & \\
\hline & & & & Placement, Diameter, Length & & & $\square$ & & $\square$ \\
\hline & & $\begin{array}{l}\text { CHW, CW, } \\
\text { HW Pipe }\end{array}$ & & $\begin{array}{l}\text { U-value, Inlet outlet water delta t, } \\
\text { Pressure drop per unit length, Pressure } \\
\text { drop by fitting, Pressure drop by plant, } \\
\text { Pressure drop by equipment/device, } \\
\text { Pressure drop by control and balancing }\end{array}$ & & & & $\square$ & \\
\hline
\end{tabular}




\begin{tabular}{|c|c|c|c|c|c|c|c|c|c|}
\hline & & & & valve & & & & & \\
\hline \multirow[b]{2}{*}{$\begin{array}{l}\text { Optimized HVAC } \\
\text { zoning }\end{array}$} & \multirow{2}{*}{$\begin{array}{l}\text { EnergyPlus, } \\
\text { eQUEST, } \\
\text { TRNSYS }\end{array}$} & AHU & & $\begin{array}{l}\text { Placement, Volume, Cooling capacity, } \\
\text { Heating capacity, Configuration, } \\
\text { Dimension }\end{array}$ & & & $\square$ & & $\square$ \\
\hline & & $\begin{array}{l}\text { Heat pump/ } \\
\text { Variable } \\
\text { Refrigerant } \\
\text { Flow (VRF) } \\
\end{array}$ & & $\begin{array}{l}\text { Placement, Heating capacity, Cooling } \\
\text { capacity, length, Configuration }\end{array}$ & & & $\square$ & & \\
\hline ECM & Simulation & Object & $\begin{array}{c}\text { Sub- } \\
\text { Object }\end{array}$ & LOD & PS & SD & $\begin{array}{l}\mathrm{D} \\
\mathrm{D}\end{array}$ & $\mathrm{CD}$ & $\begin{array}{l}\text { Cons/ } \\
\text { Ocup }\end{array}$ \\
\hline \multirow{7}{*}{ Optimized HVAC zoning } & \multirow{7}{*}{$\begin{array}{l}\text { EnergyPlus, } \\
\text { eQUEST, } \\
\text { TRNSYS }\end{array}$} & $\begin{array}{l}\text { Dedicated } \\
\text { Outdoor Air } \\
\text { System } \\
\text { (DOAS) } \\
\end{array}$ & & $\begin{array}{l}\text { Placement, Volume, Air flow rate, } \\
\text { Cooling capacity, Heating capacity }\end{array}$ & & & $\square$ & & \\
\hline & & FCU & & $\begin{array}{l}\text { Placement, Cooling capacity, Heating } \\
\text { capacity, Air flow rate, HW flow rate, } \\
\text { CHW flow rate }\end{array}$ & & & $\square$ & & $\mathbf{\square}$ \\
\hline & & Chilled beam & Diffuser & $\begin{array}{l}\text { Placement, Actuation type } \\
\text { Induction ratio, Cooling capacity, } \\
\text { Heating capacity, Air flow rate } \\
\text { HW flow rate, CHW flow rate } \\
\text { Indoor unit control }\end{array}$ & & & $\square$ & & \\
\hline & & \multirow{2}{*}{$\begin{array}{c}\text { Underfloor } \\
\text { Air } \\
\text { Distribution } \\
\text { System, } \\
\text { Displacement } \\
\text { ventilation } \\
\text { systems } \\
\end{array}$} & Diffuser & $\begin{array}{l}\text { Placement, Actuation type } \\
\text { Return air type }\end{array}$ & & & $\square$ & & $\mathbf{\square}$ \\
\hline & & & Supply fan & $\begin{array}{l}\text { Revolving type, Power, Efficiency, } \\
\text { Performance curve (Flow rate, } \\
\text { Pressure), Flow volume control, Flow } \\
\text { control }\end{array}$ & & & $\square$ & & \\
\hline & & Zone & & Placement, Area, Volume & & & $\square$ & & $\square$ \\
\hline & & Mass & & $\begin{array}{l}\text { Placement, Area, Volume, Height, } \\
\text { Orientation }\end{array}$ & & & $\square$ & & $\mathbf{\square}$ \\
\hline \multirow{3}{*}{$\begin{array}{l}\text { Heat/Enthalpy Recovery } \\
\text { Ventilation }\end{array}$} & \multirow{3}{*}{$\begin{array}{l}\text { EnergyPlus, } \\
\text { eQUEST, } \\
\text { TRNSYS }\end{array}$} & AHU & $\begin{array}{l}\text { Heat/enthalpy } \\
\text { recovery }\end{array}$ & $\begin{array}{l}\text { Type, Flow rate, Heating heat recovery } \\
\text { rate, Cooling heat recovery rate }\end{array}$ & & & & $\square$ & $\mathbf{\square}$ \\
\hline & & \multirow{2}{*}{ ERV unit } & & Placement & & & $\square$ & & $\mathbf{\square}$ \\
\hline & & & & $\begin{array}{l}\text { Type, Flow rate, Heating heat recovery } \\
\text { rate, Cooling heat recovery rate }\end{array}$ & & & & $\square$ & $\mathbf{\square}$ \\
\hline \multirow{2}{*}{$\begin{array}{l}\text { Desiccant and evaporate } \\
\text { cooling }\end{array}$} & \multirow{2}{*}{$\begin{array}{l}\text { EnergyPlus, } \\
\text { eQUEST, } \\
\text { TRNSYS }\end{array}$} & \multirow{2}{*}{ DOAS } & $\begin{array}{l}\text { Desiccant } \\
\text { Cooling }\end{array}$ & Desiccant type & & & & $\square$ & $\mathbf{\square}$ \\
\hline & & & $\begin{array}{l}\text { Evaporative } \\
\text { Cooling }\end{array}$ & $\begin{array}{lcll}\begin{array}{l}\text { Method, } \\
\text { performance curve }\end{array} & \text { rate, } & \text { Cooling } \\
\end{array}$ & & & & $\square$ & $\mathbf{\square}$ \\
\hline \multirow[b]{2}{*}{ Night purge control } & \multirow[b]{2}{*}{ EnergyPlus } & \multirow{2}{*}{$\mathrm{AHU}$} & & Night purge & & & & & \\
\hline & & & Economizer & $\begin{array}{l}\text { OA control } \\
\text { OA damper airtightness }\end{array}$ & & & & $\square$ & $\mathbf{\square}$ \\
\hline \multirow{2}{*}{$\begin{array}{l}\text { Demand Controlled } \\
\text { Ventilation(DCV) }\end{array}$} & \multirow{2}{*}{$\begin{array}{l}\text { EnergyPlus, } \\
\text { eQUEST, } \\
\text { TRNSYS } \\
\end{array}$} & $\mathrm{AHU}$ & \multirow{2}{*}{ Economizer } & \multirow{2}{*}{$\begin{array}{l}\text { OA control } \\
\text { OA damper airtightness }\end{array}$} & & & & & \\
\hline & & DOAS & & & & & & $\square$ & \\
\hline \multirow{2}{*}{$\begin{array}{c}\text { Duty cycle control } \\
\begin{array}{l}\text { Optimal on/off control } \\
\text { for AHU }\end{array} \\
\end{array}$} & \multirow{2}{*}{$\begin{array}{l}\text { EnergyPlus, } \\
\text { eQUEST, } \\
\text { TRNSYS }\end{array}$} & \multirow{2}{*}{ AHU } & & Duty cycle control & & & & $\square$ & $\mathbf{\square}$ \\
\hline & & & & Optimal on/off control & & & & $\square$ & $\mathbf{\square}$ \\
\hline \multirow{2}{*}{$\begin{array}{l}\text { Outside air temperature } \\
\text { and load reset }\end{array}$} & \multirow{2}{*}{$\begin{array}{l}\text { EnergyPlus, } \\
\text { eQUEST, } \\
\text { TRNSYS }\end{array}$} & Boiler & & HW reset & & & & $\square$ & $\mathbf{\square}$ \\
\hline & & Cooling tower & & Optimum CW temperature control & & & & $\square$ & $\mathbf{\square}$ \\
\hline $\begin{array}{c}\text { Garage Carbon } \\
\text { Monoxide control }\end{array}$ & TRNSYS & AHU & & $\mathrm{CO}$ control for garage & & & & $\square$ & $\mathbf{\square}$ \\
\hline \multirow{2}{*}{$\begin{array}{l}\text { High efficiency water } \\
\text { heater }\end{array}$} & \multirow{2}{*}{$\begin{array}{l}\text { EnergyPlus, } \\
\text { eQUEST, } \\
\text { TRNSYS }\end{array}$} & \multirow{2}{*}{ Water heater } & & Placement, Hot water capacity & & & $\square$ & & $\mathbf{\square}$ \\
\hline & & & & Fuel type, Efficiency, Water storage & & & & $\square$ & $\mathbf{\square}$ \\
\hline Insulated pipe & $\begin{array}{l}\text { EnergyPlus, } \\
\text { eQUEST, } \\
\text { TRNSYS } \\
\end{array}$ & $\begin{array}{l}\text { CHW, CW, } \\
\text { HW, DHW } \\
\text { pipe }\end{array}$ & & U-value & & & & $\square$ & $\mathbf{\square}$ \\
\hline \multirow{2}{*}{$\begin{array}{l}\text { Water saving closet and } \\
\text { tab }\end{array}$} & \multirow{2}{*}{$\begin{array}{l}\text { EnergyPlus, } \\
\text { Equest } \\
\text { *only water } \\
\text { heating }\end{array}$} & & & Placement, Volume, Heat & & & & $\square$ & $\mathbf{\square}$ \\
\hline & & Closet, Tab & & $\begin{array}{l}\text { Boiling method } \\
\text { Insulation }\end{array}$ & & & & $\square$ & $\mathbf{\square}$ \\
\hline Daylight sensor & $\begin{array}{l}\text { EnergyPlus, } \\
\text { eQUEST, } \\
\text { TRNSYS }\end{array}$ & Ambient light & & $\begin{array}{l}\text { Light schedule, Daylight control, LED } \\
\text { deeming control, Exterior light } \\
\text { automatic on/off, Grouping control }\end{array}$ & & & & $\square$ & $\mathbf{\square}$ \\
\hline $\begin{array}{l}\text { Deeming and on/off } \\
\text { control }\end{array}$ & $\begin{array}{l}\text { EnergyPlus, } \\
\text { eQUEST, } \\
\text { TRNSYS }\end{array}$ & Ambient light & & $\begin{array}{l}\text { Light schedule, LED deeming control, } \\
\text { Exterior light automatic on/off, } \\
\text { Grouping control }\end{array}$ & & & & $\square$ & $\mathbf{\square}$ \\
\hline
\end{tabular}




\begin{tabular}{|c|c|c|c|c|c|c|c|c|c|}
\hline ECM & Simulation & Object & $\begin{array}{c}\text { Sub- } \\
\text { Object }\end{array}$ & LOD & PS & SD & $\begin{array}{l}\mathrm{D} \\
\mathrm{D}\end{array}$ & $\mathrm{CD}$ & $\begin{array}{l}\text { Cons/ } \\
\text { Ocup }\end{array}$ \\
\hline $\begin{array}{l}\text { Lighting schedule per } \\
\text { space }\end{array}$ & $\begin{array}{l}\text { EnergyPlus, } \\
\text { eQUEST, } \\
\text { TRNSYS }\end{array}$ & Ambient light & & Light schedule & & & & $\square$ & \\
\hline \multirow{5}{*}{$\begin{array}{l}\text { Photovoltaic exterior } \\
\text { light }\end{array}$} & \multirow{5}{*}{$\begin{array}{l}\text { EnergyPlus, } \\
\text { eQUEST, } \\
\text { TRNSYS }\end{array}$} & Ext. light & & $\begin{array}{l}\text { Placement, Wattage, On-site PV } \\
\text { attached }\end{array}$ & & & & $\square$ & \\
\hline & & \multirow{4}{*}{ Photovoltaic } & & $\begin{array}{l}\text { Placement, Area, Azimuth, Angle, } \\
\text { Seasonal shade }\end{array}$ & & & & $\square$ & \\
\hline & & & Solar panel & $\begin{array}{l}\text { Module type, Tracking mode } \\
\text { Generation capacity, Generation } \\
\text { efficiency } \\
\text { Nominal operation cell temperature, } \\
\text { Temperature coefficient, Loss } \\
\text { coefficient }\end{array}$ & & & & $\square$ & \\
\hline & & & \multirow{2}{*}{ Inverter } & Placement, Capacity & & & & $\square$ & $\square$ \\
\hline & & & & Efficiency & & & & $\square$ & \\
\hline Parasitic load control & $\begin{array}{l}\text { EnergyPlus, } \\
\text { eQUEST, } \\
\text { TRNSYS } \\
\end{array}$ & $\begin{array}{l}\text { Static } \\
\text { capacitor }\end{array}$ & & Placement, Voltage, Wattage, Current & & & & $\square$ & \\
\hline \multirow{2}{*}{$\begin{array}{l}\text { Sequence control, group } \\
\text { and schedule } \\
\text { management } \\
\text { of elevator/escalator }\end{array}$} & \multirow{2}{*}{$\begin{array}{l}\text { EnergyPlus, } \\
\text { eQUEST, } \\
\text { TRNSYS }\end{array}$} & \multirow[b]{2}{*}{$\begin{array}{l}\text { Elevator/ } \\
\text { Escalator }\end{array}$} & & Placement, Number & & & $\square$ & & $\square$ \\
\hline & & & & Control & & & & $\square$ & \\
\hline \multirow{9}{*}{$\begin{array}{l}\text { Solar water and space } \\
\text { heating }\end{array}$} & \multirow{9}{*}{$\begin{array}{l}\text { EnergyPlus, } \\
\text { TRNSYS }\end{array}$} & \multirow{3}{*}{ Solar panel } & & $\begin{array}{l}\text { Placement, Area, Azimuth, Altitude, } \\
\text { Seasonal shade }\end{array}$ & & $\square$ & & & \\
\hline & & & & Type & & & $\square$ & & \\
\hline & & & & Capacity, Efficiency, Absorptivity & & & $\square$ & & \\
\hline & & \multirow{2}{*}{$\begin{array}{l}\text { Hot water } \\
\text { tank }\end{array}$} & & Placement, Volume, Heat capacity & & & $\square$ & & \\
\hline & & & & Insulation & & & & $\square$ & \\
\hline & & \multirow{3}{*}{ Primary pump } & & Placement, Power & & & $\square$ & & \\
\hline & & & & $\begin{array}{l}\text { Efficiency, Performance curve (Flow } \\
\text { rate, Head) }\end{array}$ & & & $\square$ & & \\
\hline & & & & Flow control & & & & $\square$ & \\
\hline & & $\begin{array}{c}\text { Heat } \\
\text { exchanger }\end{array}$ & & Placement, Capacity, Efficiency & & & $\square$ & & \\
\hline \multirow{8}{*}{ Solar air heater } & \multirow{8}{*}{$\begin{array}{l}\text { EnergyPlus, } \\
\text { TRNSYS }\end{array}$} & \multirow{3}{*}{ Solar panel } & & $\begin{array}{l}\text { Placement, Area, Azimuth, Altitude, } \\
\text { Seasonal shade }\end{array}$ & & $\square$ & & & \\
\hline & & & & Type & & & $\square$ & & \\
\hline & & & & $\begin{array}{l}\text { Capacity, Efficiency, Absorptivity, } \\
\text { Flow volume }\end{array}$ & & & $\square$ & & \\
\hline & & \multirow{8}{*}{ Heat pump } & & Placement, Power & & & $\square$ & & $\square$ \\
\hline & & & & Revolving type & & & $\square$ & & $\square$ \\
\hline & & & & $\begin{array}{l}\text { Efficiency, Performance curve (Flow } \\
\text { rate, Pressure) }\end{array}$ & & & & $\square$ & \\
\hline & & & & $\begin{array}{l}\text { Flow volume control } \\
(\mathrm{CAV}, \mathrm{VAV})\end{array}$ & & & & $\square$ & \\
\hline & & & & $\begin{array}{l}\text { Variable flow control } \\
\text { (RPM, Outlet/Inlet damper, Inlet vane, } \\
\text { Variable pitch }\end{array}$ & & & & $\square$ & \\
\hline \multirow{10}{*}{ Geothermal heat pump } & \multirow{10}{*}{$\begin{array}{l}\text { EnergyPlus, } \\
\text { eQUEST, } \\
\text { TRNSYS }\end{array}$} & & & Placement & & $\square$ & & & \\
\hline & & & & $\begin{array}{l}\text { Cooling capacity, Heating capacity, } \\
\text { Cooling COP, Heating COP }\end{array}$ & & & $\square$ & & \\
\hline & & & & Compressor control & & & & $\square$ & \\
\hline & & Ground heat & & $\begin{array}{l}\text { Placement, Land area, Length, } \\
\text { Number, Pipe diameter, Distance } \\
\text { between pipes }\end{array}$ & & $\square$ & & & \\
\hline & & exchanger & & Type & & $\square$ & & & $\mathbf{\square}$ \\
\hline & & & & Capacity, Grouting conductivity & & & $\square$ & & $\square$ \\
\hline & & & & Placement, Power & & & $\square$ & & $\square$ \\
\hline & & $\begin{array}{l}\text { Primary and } \\
\text { Secondary }\end{array}$ & & $\begin{array}{l}\text { Efficiency, Performance curve (Flow } \\
\text { rate, Head) }\end{array}$ & & & $\square$ & & \\
\hline & & & & Flow control & & & & $\square$ & $\square$ \\
\hline & & $\begin{array}{l}\text { Expansion } \\
\text { tank }\end{array}$ & & Placement, Volume, Heat capacity & & & $\square$ & & \\
\hline
\end{tabular}


TABLE II: PHASES OF THE BUILDING DESIGN PROCESS

\begin{tabular}{|c|c|}
\hline Phase & Detailed tasks \\
\hline $\begin{array}{l}\text { Pre-Schematic } \\
\text { Design (PS) }\end{array}$ & $\begin{array}{l}\text { Outline space plan, Check Building code, } \\
\text { Field study, Analysis of existing building, } \\
\text { User analysis }\end{array}$ \\
\hline $\begin{array}{c}\text { Schematic } \\
\text { Design (SD) }\end{array}$ & $\begin{array}{c}\text { Rough estimation, Planning of building and } \\
\text { MEP, Examine approval process }\end{array}$ \\
\hline $\begin{array}{c}\text { Design } \\
\text { Development (DD) }\end{array}$ & $\begin{array}{c}\text { Approval, } \\
\text { Drawing layer } \\
\end{array}$ \\
\hline $\begin{array}{c}\text { Construction } \\
\text { Document (CD) }\end{array}$ & $\begin{array}{c}\text { Embody drawing, } \\
\text { Create construction document, Estimating } \\
\text { detailed construction expenses }\end{array}$ \\
\hline $\begin{array}{l}\text { Construction/ } \\
\text { Post occupancy } \\
\text { (Cons/Ocup) }\end{array}$ & $\begin{array}{l}\text { Bidding, } \\
\text { Construction, Operation }\end{array}$ \\
\hline
\end{tabular}

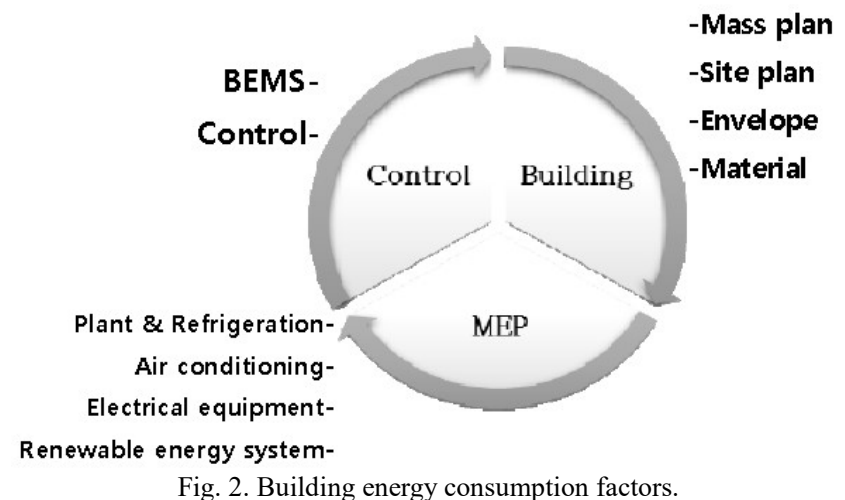

\section{LEVEL OF DETAIL (LOD) OF AN ECM}

First, we have investigated the design phases at which design elements and attributes of architectural and MEP objects are determined according to the building design process in Table III.

TABLE III: PRIMARy Design ENTITIES Determined IN EACH Design PHASE

\begin{tabular}{|c|c|c|}
\hline Design phase & Architecture & MEP \\
\hline $\begin{array}{l}\text { Pre-Schematic } \\
\text { Design } \\
\text { (PS) }\end{array}$ & $\begin{array}{c}\text { Mass plan, } \\
\text { Standards for design }\end{array}$ & $\begin{array}{l}\text { Placement, Type and } \\
\text { area of Geothermal } \\
\text { heat exchange }\end{array}$ \\
\hline $\begin{array}{l}\text { Schematic Design } \\
\text { (SD) }\end{array}$ & $\begin{array}{l}\text { Building size, } \\
\text { Zoning, Shape, } \\
\text { Material }\end{array}$ & $\begin{array}{c}\text { Schedule, } \\
\text { Block planning of } \\
\text { Renewable system }\end{array}$ \\
\hline $\begin{array}{c}\text { Design } \\
\text { Development (DD) }\end{array}$ & $\begin{array}{l}\text { Detailed } \\
\text { Property }\end{array}$ & $\begin{array}{c}\text { Placement, Type, } \\
\text { Capacity, Efficiency, } \\
\text { Control }\end{array}$ \\
\hline $\begin{array}{l}\text { Construction } \\
\text { Document } \\
\text { (CD) }\end{array}$ & Fixed & Fixed \\
\hline
\end{tabular}

In the pre-schematic design phase, layout and mass planning (such as location, orientation, height and area of a building) are mainly discussed with a feasibility study and then confirmed in the schematic design phase. Design criteria and conditions are already fixed (such as the surrounding terrain and climate of the building) before the design process begins. For MEP, it is very little to be determined at this stage because no specific design values are available yet. Since a ground heat exchanger is, however, installed in the site, the position and area of the geothermal system need to be considered together within the building layout.

In the schematic design stage, an overall shape, structure and materials of a building (such as envelopes, story height, stairs) are determined. In the MEP, user schedule, lighting and ventilation can be captured considering the use and size of the building. In addition, properties such as location and area are discussed for design entities located outside the building such as solar panels or underground heat exchangers.

In the design development stage, as most design values related to the building is determined, properties of MEP are started to be determined. At the beginning of this phase, placement, type and capacity of the equipment are settled down and properties related to the building controls are determined in the latter part of this stage.

In the construction document phase, most properties are determined and there can be necessary design changes. In Table I, the design phase at which each ECM is discussed is marked by $\square$, and the design phase at which it is confirmed is indicated by

\section{Simulation PROgRAMS For EVALUATING BUILDING ENERGY PERFORMANCE}

Building energy simulation has been developed since 1970, and a use of simulation tools has been highly encouraged for green building designs. However, contrary to the advocacy group who goes for a new technology of building simulation, the pragmatist groups such as design engineers are somehow reluctant to understand unfamiliar simulations.

Therefore, in this paper, we have tried to propose a guideline of simulation use in order to encourage the simulation practitioners in easily evaluating the building energy performance. We have investigated a functionality of DOE-2.1, ECOTECT, EnergyPlus, eQUEST, ESP-r, HAP, IDA ICE, IES $<\mathrm{VE}>$, Tas, TRACE and TRNSYS to determine what simulation tools best capture ECMs at each design stage. But only EnergyPlus, eQUEST and TRNSYS are marked in Table I, because they are mostly used simulations in Korea.

\section{CONCLUSION}

To encourage energy simulations in building design, this paper proposes a guideline concerning what simulation is appropriate for each ECM to practitioner designers who are lack of the expertise in energy simulation. We hope to convince the people who have a sense of discomfort with unfamiliar simulations, such that various applications of building energy simulation can be tried out in the design phase. We also expect a convergence between architectural designers who is lack of the expertise of equipment, and MEP designers who is lack of the expertise of architecture. Therefore a systematic and integrated design can be implemented from the initial planning phase to the operation and maintenance phase.

\section{ACKNOWLEDGEMENT}

This research was supported by Basic Science Research Program through the National Research Foundation of Korea (NRF) funded by the Ministry of Science, ICT \& Future 


\section{Planning (NRF-2015R1C1A2A01053559).}

\section{REFERENCES}

[1] S. H. Kim, "How to improve usability of building energy simulation for the integrated design process," KIEAE Journal, vol. 15, no. 6, pp. 47-56, Dec. 2015

[2] S. H. Kim, "Level of detail (LOD) for building energy conservation measures (ECMs)," KIEAE Journal, vol. 15, no. 6, pp. 69-81, Dec. 2015

[3] D. W. Kim and C. S. Park, "Needs and issues for better use of building energy simulation tools at design stage," Journal of the AKI, vol. 28, no. 10 , pp. $317-325$, Oct. 2012

[4] J. Park, Y. S. Kang, and P. C. Ihm, "Development of simplified building energy simulation program for building energy performance Analysis," REF-J, vol. 21, no. 1, pp. 9-15, Jan. 2009.

[5] M. Oh, J. H. Ahn, and J. W. Lee, "A proposal of LOD for the domestic BIM-based MEP design management," in Proc. KICEM, 2013, pp. 311-333.

[6] S. Y. Choo, K. H. Lee, and S. K. Park, "A study on LOD for development of Green BIM guidelines," Journal of the AIK, vol. 28, no. 6, pp. 37-47, Jun. 2012

[7] Y. D. Kim, "Use of building energy simulation program for energy efficient and sustainable building design," KARSE Magazine, vol. 26, no. 9 , pp. 65-85, Sept. 2009

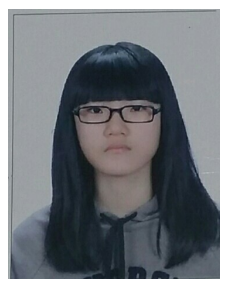

Mina Choi will receive a B.S degree in engineering in February 2017 from the School of Architecture of Seoul National University of Science and Technology, Seoul, South Korea.

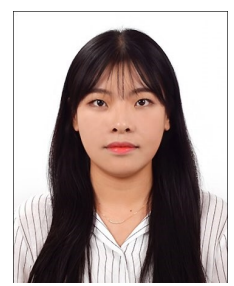

Gahee Kim will receive a B.S degree in engineering in February 2017 from the School of Architecture of Seoul National University of Science and Technology, Seoul, South Korea.

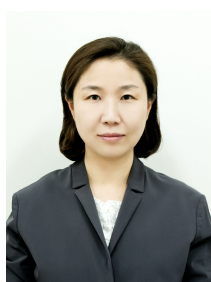

Sean Hay Kim received a Ph.D. from the Georgia Tech. Before joining the Seoul National University of Science and Technology, she worked for Siemens and Autodesk in the U.S. 